\title{
High dose naltrexone for dyskinesias induced by levodopa
}

\author{
A J Manson, R Katzenschlager, J Hobart, A J Lees
}

\begin{abstract}
Ten patients with Parkinson's disease and levodopa induced dyskinesias (LIDs) took part in this randomised, placebo controlled, double blind, crossover trial to assess the efficacy and tolerability of high dose oral naltrexone for LIDs in Parkinson's disease. Patients received naltrexone (5 $\mathrm{mg} / \mathrm{kg} /$ day) or placebo for 2.5 weeks with 1 week wash out in between. Dyskinesias and motor function were assessed with a levodopa challenge, unified Parkinson's disease rating scale (UPDRS), the unified dyskinesia rating scale (UDRS), and patient diaries. Eight patients completed the trial. There was a small reduction in LIDs measured by patient diaries with naltrexone $(20.5$ (SD 24.9$) \%$ ) compared with placebo $(-4.1$ (SD 22.6)\%), p <0.05, although no difference was found by other subjective or objective measures. Naltrexone was well tolerated and caused no significant differences in UPDRS motor scores or off time. This study suggests that short term therapy with high dose naltrexone (250$350 \mathrm{mg} /$ day) has no or minimal effect on reducing LIDs in Parkinson's disease.

(F Neurol Neurosurg Psychiatry 2001;70:554-556)
\end{abstract}

Keywords: naltrexone; levodopa induced dyskinesias; Parkinson's disease

Levodopa induced dyskinesias (LIDs) are a considerable challenge in the long term management of Parkinson's disease. Recently, nondopaminergic pathways have been targeted as a means of controlling dyskinesias without worsening parkinsonism, ${ }^{1}$ and promising results with the glutamate antagonist amantadine have been reported. ${ }^{23}$

Levodopa induced dyskinesias are thought to be associated with changes in the balance of neurotransmitter systems within the basal ganglia, ${ }^{4}$ and it is thought that opioid receptor antagonism may reverse some of these changes and thus lessen dyskinesias. ${ }^{1}$ Conflicting results with opiate receptor antagonists for LIDs in Parkinson's disease have been previously published. Acute intravenous administration of naloxone successfully reduced LIDs in two out of three studies ${ }^{56}$ whereas $100 \mathrm{mg} /$ day naltrexone, an orally active opiate antagonist licensed for use in opiate addiction, was ineffective. ${ }^{7}$
However, recent studies with the MPTP lesioned marmoset model of Parkinson's disease demonstrated a marked reduction of LIDs with oral naltrexone at doses of $10 \mathrm{mg} / \mathrm{kg} /$ day. ${ }^{18}$ The aim of this study was to investigate the antidyskinetic effect of higher dose naltrexone $(5 \mathrm{mg} / \mathrm{kg} /$ day $)$.

\section{Methods}

Ten patients (six men and four women) with idiopathic Parkinson's disease participated in the trial. Their mean age was 62 (range 53-80) years, mean duration of Parkinson's disease, 13.2 (range 8-22) years, and mean duration of levodopa therapy, 11.5 (range 7-17) years. Six patients were taking oral dopamine agonists (mean pergolide equivalent dose $3.2 \mathrm{mg} /$ day), one was on a continuous apomorphine infusion, and two were taking amantadine. All had disabling LIDs and had been receiving a fixed dose of their usual antiparkinsonian medication for a period of at least 1 month before inclusion. Exclusion criteria included patients with moderate to severe hepatic impairment, concurrent use of opioid containing medication or opiate dependency, hypersensitivity to naltrexone, and moderate to severe dementia. All patients gave informed consent to participate and the joint medical ethics committee of the National Hospital for Neurology and Neurosurgery approved the study.

Baseline screening tests, performed between 1 and 2 weeks before the start of the study, included a full medical history and examination, mini mental state examination, ${ }^{9}$ an ECG, full blood count, urea, electrolytes, and liver function testing. The trial was double blind, placebo controlled, and crossover in design with 2.5 weeks on each treatment separated by 1 week for washout. Patients were given oral naltrexone ( $5 \mathrm{mg} / \mathrm{kg}$ (to the nearest $50 \mathrm{mg}$ )), in three divided doses, to be taken after meals. The dose was gradually increased from $100 \mathrm{mg}$ by $50-100 \mathrm{mg}$ increments a day over the 3 to 4 days of each treatment period.

Patients were assessed with levodopa challenges, at baseline, and at the end of each treatment period. The challenges were performed in a standard fashion using the patient's normal maximum dose (range 100-300 mg) required to achieve the on state and assessed after an overnight fast and withdrawal of medication (except naltrexone), using Hoehn and Yahr 
ratings and unified Parkinson's disease rating scale (UPDRS) in the off and full on state. Once the on state was achieved, patients were videotaped three times at 20 minute intervals. During each recording, patients were engaged in the following motor and mental tasks, previously shown to elicit dyskinesia ${ }^{10}{ }^{11}$ :

(1) Sitting still for 1 minute at rest

(2) Mental calculation

(3) Drinking from a cup

(4) Putting on and buttoning a coat

(5) Walking.

At the end of the challenge, patients were also videotaped preparing and eating breakfast.

Dyskinesias were assessed blindly by two trained neurologists (AJM and RK) on later review of the videotape, using the modified Goetz 5 point $(0-4)$ severity scale ${ }^{12}$ for tasks 3-5 (excluding phenomenological rating) and the modified AIM scale, for task 1 and 2. ${ }^{113}$ Orofacial and buccolingual, global, and dental ratings were excluded from the AIM scale, giving a maximum score of 24 . Both scales were used to rate dyskinesias while preparing and eating breakfast.

Patients were also asked to complete diary cards for five days and the Lang and Fahn unified dyskinesia rating scale (UDRS) ${ }^{14}$ before each assessment. The diaries included daily recording of time spent in the on and off states, and an overall daily subjective dyskinesia assessment using a visual analogue scale (VAS). After each treatment phase, patients were also asked about their subjective impression of dyskinesia improvement and adverse effects on a four point scale $(0=$ none, $1=$ mild, $2=$ moderate, $3=$ marked).

Patients were given prerandomised treatment numbers in the order in which they entered the trial, receiving blindly naltrexone (50 mg capsules) or matched placebo first.

For the objective video ratings, mean Goetz and AIM scores were taken for each assessment for each rater and the mean of the two raters' scores was then taken for the final analysis. Results for the two scales were analysed separately.

For the subjective diary ratings of dyskinesia severity and off time, the mean VAS score (measured in $\mathrm{cm}$ ), and mean off time (as a percentage of the waking day) from the 5 days scored was taken for each patient.

Each patient's percentage reduction in dyskinesia score from baseline was calculated separately for both treatment arms and the means of the percentage reduction for the two treatment arms were compared using Wilcoxon's test.

Interrater reliability was assessed by first correlating the two raters' scores and then assessing the mean difference of each rater's score from the mean of the two raters' scores for each assessment.

\section{Results}

Eight patients completed the trial. One patient dropped out due to adverse effects on day 1 of the first treatment period. Another dropped out before the first treatment phase due to a psychotic episode during baseline assessment. Neither patient was included in the final analysis. The mean dose of naltrexone taken was 306 (range 250 - 350) $\mathrm{mg} /$ day.

The results are summarised in table 1 .

Interrater reliability for the blinded objective dyskinesia rating was good for both scales. Pearson's $r$ between the two raters' scores for AIMS was $0.86 \quad(p<0.01)$, with a mean difference between scores of 0.6 (SD 0.5 ). Spearman's $r$ for the Goetz scale was 0.67 $(\mathrm{p}<0.01)$, with a mean difference between raters' scores of 0.3 (SD 0.2).

No change in daily on times or objective UPDRS scores were found between the two treatment periods.

Severe nausea and vomiting led to withdrawal on the first day of naltrexone treatment in one patient. The patient had inadvertently taken $350 \mathrm{mg}$ naltrexone, without titrating up the dose, on an empty stomach. Two further patients reported moderate to severe anorexia during the naltrexone treatment period but were able to continue. Naltrexone was otherwise well tolerated and there were no changes in liver function. There were no adverse events during the placebo phase.

\section{Discussion}

A very mild subjective improvement in dyskinesia occurred with naltrexone $(5 \mathrm{mg} / \mathrm{kg} /$ day). No improvement was seen with objective measures, and only two patients reported a moderate improvement of dyskinesias with naltrexone, which was not different from placebo. Although the patient numbers were small, this study was designed to investigate a clinically relevant antidyskinetic effect, which has been shown to be possible by careful and detailed analysis of small samples. ${ }^{3} 15$

Table 1 Effect of high dose naltrexone and placebo on dyskinesia by all measures

\begin{tabular}{|c|c|c|c|c|}
\hline Measure & $\begin{array}{l}\text { Baseline Mean } \\
\text { (range/SD) }\end{array}$ & $\begin{array}{l}\text { Placebo Mean } \\
\text { (range/SD) }\end{array}$ & $\begin{array}{l}\text { Naltrexone Mean } \\
\text { (range/SD) }\end{array}$ & $\begin{array}{l}P \text {-value for } \\
\text { difference }\end{array}$ \\
\hline \multicolumn{5}{|l|}{ Objective dyskinesia ratings: } \\
\hline Mean AIM scores (max 24$)$ & $9.3(4.8-12.7)$ & $10.2(7.25-13.1)$ & $9.8(7-14.8)$ & \\
\hline Mean $\%$ reduction Aims scores & & $-13.4(20.1)$ & $-9.4(22.1)$ & 0.7 \\
\hline Mean Goetz scores $(\max 4)$ & $1.5(0.8-2.4)$ & $1.5(1.0-2.1)$ & $1.5(1.0-2.0)$ & \\
\hline Mean \% reduction Goetz scores & & $0.003(0.002)$ & $0.007(0.1)$ & 0.4 \\
\hline \multicolumn{5}{|l|}{ Subjective impression: } \\
\hline Diary scores & $5.5(3.9-7.7)$ & $5.51(3.5-8.3)$ & $4.4(2.7-8.4)$ & \\
\hline Mean $\%$ reduction diary scores & & $-4.1(22.5)$ & $20.5(24.9)$ & 0.03 \\
\hline Mean UDRS score & $12.1(10-16)$ & $1.7(1.0)$ & $0.3(0.3)$ & \\
\hline Mean $\%$ reduction UDRS scores & & $9.7(18.7)$ & $11.2(16.3)$ & 0.7 \\
\hline Mean \% reduction UPDRS item 32 (dyskinesia severity) & & $9.4(18.1)$ & $12.5(35.4)$ & 0.7 \\
\hline Mean $\%$ reduction UPDRS item 33 (dyskinesia duration) & & $0(26.7)$ & $18.7(37.2)$ & 0.4 \\
\hline Patient impression ( 4 point scale) & & $0.3(0.5)$ & $0.6(0.9)$ & 0.3 \\
\hline
\end{tabular}

Results are means (data ranges or SD as appropriate) 
Naltrexone may exert a very small antidyskinetic effect, which could only be detected by patient diaries. As these do not have a standardised scale, but work via VASs, based on individual patients' severity ranges, they are potentially more sensitive. However, it seems improbable that an important clinically relevant antidyskinetic effect has been missed.

Several non-dopaminergic drugs, including opioid antagonists, have been reported to have potent antidyskinetic effects in animal models, ${ }^{1816}$ and this study illustrates the difficulty in translating these results to clinical practice. A possible explanation for this discrepancy could be the relatively lower dose of naltrexone ( $5 \mathrm{mg} / \mathrm{kg} /$ day) used in our study, compared with $10 \mathrm{mg} / \mathrm{kg} /$ day used in the MPTP lesioned marmosets. However, the dosage used was the maximum allowed by our centre's ethics committee, due to concerns about increases in serum transaminases. ${ }^{17}$ Doses up to 800 $\mathrm{mg} /$ day have, however, been shown to be well tolerated and non-toxic in volunteers and clinical trials in psychiatric disorders, and long term treatment for opiate addiction with $350 \mathrm{mg}$ /day has proved safe. ${ }^{18}$

Naltrexone is active at $\mu, \kappa$, and $\delta$ receptors, and antagonism at $\kappa$ and $\delta$ opioid receptors could potentially reduce LIDs through modulation of the direct and indirect striatopallidal pathways, as previously described. However, naltrexone is preferentially active at $\mu$ receptors, ${ }^{19}$ and although these have been implicated in LID generation, ${ }^{8}$ the selective $\mu$ antagonist cyprodime failed to suppress involuntary movements in the rat model of LID. ${ }^{20}$ It is therefore possible that naltrexone's activity at $\delta$ receptors at the dosage used is inadequate to attenuate dyskinesia.

Although the previous clinical and preclinical studies successfully demonstrating an antidyskinetic effect did so acutely, it is conceivable that resetting of the receptors may require longer treatment periods. Reduction of dyskinesia with apomorphine monotherapy usually takes 3 to 6 months. ${ }^{21}$

Studies with higher doses of naltrexone or for longer treatment periods may therefore be warranted.

We thank Dupont Pharma for the supply of Naltrexone, Mike O'Sullivan for helping with the video challenges, and The Reta Lila Weston Institute for funding.
1 Brotchie JM. Adjuncts to dopamine replacement: a pragmatic approach to reducing the problem of dyskinesia in Parkinson's disease. Mov Disord 1998;13:871-6.

2 Verhagen Metman L, Del Dotto P, van den Munckhof P, et al. Amantadine as treatment for dyskinesias and motor fluctuations in Parkinson's disease [see comments]. Neurology 1998;50:1323-6.

3 Luginger E, Wenning GK, Bosch S, et al. Beneficial effects of amantadine on L-dopa-induced dyskinesias in Parkinson's disease [[in process citation]]. Mov Disord 2000;15: son's disea $873-8$.

4 Crossman AR. A hypothesis on the pathophysiological mechanisms that underlie levodopa- or dopamine agonistinduced dyskinesia in Parkinson's disease: implications for future strategies in treatment. Mov Disord 1990;5:100-8.

5 Sandyk R, Snider SR. Naloxone treatment of L-dopainduced dyskinesias in Parkinson's disease [[letter]]. Am f Psychiatry 1986;143:118

6 Trabucchi M, Bassi S, Frattola L. Effect of naloxone on the "on-off" syndrome in patients receiving long-term levodopa therapy. Arch Neurol 1982;39:120-1.

7 Rascol O, Fabre N, Blin O, et al. Naltrexone, an opiate antagonist, fails to modify motor symptoms in patients with Parkinson's disease. Mov Disord 1994;9:437-40.

8 O'Byrne MB, Henry B, Crossman AR, et al. The role of opioid peptides in L-DOPA-induced dyskinesias in parkinsonism. Mov Disord 1998;13(suppl 2):P2.033.

9 Folstein MF, Folstein SE, McHugh PR. Mini-mental state. A practical method for grading the cognitive state of patients for the clinician. $\mathcal{F}$ Psychiatr Res 1975;12:189-98.

10 Durif F, Vidailhet M, Debilly B, et al. Worsening of levodopa-induced dyskinesias by motor and mental tasks [[in process citation]]. Mov Disord 1999;14:242-5.

11 Manson AJ, Brown P, O'Sullivan JD, et al. An ambulatory dyskinesia monitor. $\mathcal{F}$ Neurol Neurosurg Psychiatry 2000;68: 196-201.

12 Goetz CG, Stebbins GT, Shale HM, et al. Utility of an objective dyskinesia rating scale for Parkinson's disease: inter- and intrarater reliability assessment [[see comments]]. Mov Disord 1994;9:390-4.

3 May PR, Lee MA, Bacon RC. Quantitative assessment of neuroleptic-induced extrapyramidal symptoms: clinical and nonclinical approaches. Clin Neuropharmacol 1983; 6(suppl 1):S35-51

14 Nutt A. Unified dyskinesia rating scale for dyskinesias? Mov Disord 1999;14(suppl 1):74-80.

15 Manson AJ, Schrag A, Lees AJ. Low-dose olanzapine for levodopa induced dyskinesias [[in process citation]]. Neurology 2000;55:795-9.

16 Henry B, Fox SH, Peggs D, et al. The $\alpha 2$-adrenergic receptor antagonist Idazoxan reduces dyskineia and enhances anti-parkinsonian actions of 1-dopa in the MPTP-lesioned primate model of Parkinson's disease. Mov Disord 1999;14: primate

17 Kosten TR, Kreek MJ, Ragunath J, et al. Cortisol levels during chronic naltrexone maintenance treatment in ex- opiate addicts. Biol Psychiatry 1986;21:217-20.

18 Brahen LS, Capone TJ, Capone DM. Naltrexone: lack of effect on hepatic enzymes. F Clin Pharmacol 1988;28:6470.

19 Vermeulen RJ, Drukarch B, Sahadat MC, et al. Morphine and naltrexone modulate D2 but not D1 receptor induced motor behavior in MPTP-lesioned monkeys. Psychopharmacology (Berl) 1995;118:451-9.

20 Henry B, Crossman A, Brotchie JM. Opioid peptide involvement in L-DOPA-induced dyskinesias: molecular and behavioural studies following long-term tretment in the 6-OHDA-lesioned rat model of Parkinson's disease [[abstract]]. Mov Disord 1996;11(suppl 1):61.

21 Colzi A, Turner K, Lees AJ. Continuous subcutaneous waking day apomorphine in the long term treatment of levodopa induced interdose dyskinesias in Parkinson's disease. F Neurol Neurosurg Psychiatry 1998;64:573-6. 